\title{
A Timestamp Tree-Based Cache Invalidation Report Scheme in Mobile Computing Environments ${ }^{\star}$
}

\author{
Hakjoo Lee ${ }^{1}$, Jonghyun Suh², Sungwon Jung ${ }^{2}$, \\ Sooyoung Lee ${ }^{3}$, and Junguck Lee ${ }^{3}$ \\ ${ }^{1}$ DTV Lab. AIM Gr., LG Electronics, San 4-1, \\ Bongcheon 7, Gwanak-gu, Seoul, Korea \\ or4nge@lge.com \\ 2 Department of Computer Science, Sogang University, Seoul, Korea \\ \{joe77, jungsung\}@sogang.ac.kr \\ ${ }^{3}$ R\&D Division, Advanced Power\&IT Research Center, KATECH, Korea
}

\begin{abstract}
In mobile computing environments, cache invalidation techiniques are widely accepted. However, theses techniques require a large size of the invalidation report and show low cache utilization under high server update rate. We propose a new cache-level cache invalidation technique called TTCI(Timestamp Tree-based Cache Invalidation technique) to overcome the above problems. TTCI also supports selective tuning for a cache-level cache invalidation. We verify the effectiveness of our TTCI schemes through in-depth simulation experiments.
\end{abstract}

\section{Introduction}

In mobile computing environments, cache invalidation techiniques are widely accepted. But it is very difficult due to frequent disconnections and relatively unreliable wireless links. There have been many research efforts to develop efficient cache-level cache invalidation report schemes for mobile clients [3, 4. However, under high update rate, invalidation report size increases proportionally and false invalidation will occur more often which causes low cache utilization. And none of the above schemes support selective tuning, which is very important for mobile clients to conserve the scarce battery resources. There have been other efforts like [2. However, it lacks scalability and consumes more bandwidth including uplink. To cope with the above problems, we propose a new cache invalidation report scheme called Timestamp Tree-based Cache Invalidation(TTCI) without using any uplink. Also unlike group-based IR and BS-based IR schemes, TTCI doesn't require any priori knowledge.

\footnotetext{
* This research was supported in part by grant No.10016775 under the supervision of Korea Automotive Technology Institute from the next-generation new growth engine program of Ministry Of Commerce and Industry and Energy in Korea and in part by grant No.R01-2003-000-10197-0 from the Basic Research Program of the Korea Science\&Engineering Foundation.
} 
The rest of the paper is organized as follows. In Section 2 and 3, we describe our TTCI scheme and give performance analysis through simulation experiments. Finally, Section 4 provides concluding remarks.

\section{Timestamp Tree-Based Cache Invalidation(TTCI)}

\subsection{TTCI Algorithm}

In this subsection, we introduce the TTCI algorithm using an example. Basically, TTCI is composed of TT(Timestamp Tree) and IS(ID liSt). TT is a timestampbased tree and works like an index helping clients to find a proper location of the updated information. IS is a list of the updated data IDs. Clients listen to the updated data from the position where TT points till the end of IS.

Suppose there are 20 updated data with $N T=16$ non-identical timestamps, $F A=3$ fanout of TT, and $N T S=8$ the number of timestamps that TT uses. TTCI works in four steps as follows: First, build OLIST, a list of the updated data IDs sorted by timestamps in ascending order with no duplications, as shown in Table 1. Second, using timestamps from OLIST, build TT. A node in TT is composed of pointers $\left(P_{i}\right)$ and timestamps $\left(T S_{i}\right)$, like $<P_{1}, T S_{1}, P_{2}, T S_{2}, P_{3}$, $T S_{3}, \ldots, T S_{F A-1}, P_{F A}>$. This step is described in Algorithm 11, Third, build IS, which is a list of the updated data IDs in order of timestamps. We simply sort OLIST by timestamps. Finally, combine IS and TT. Pointers in the internal nodes are set pointing to another node and pointers in the leaf nodes are set pointing to a proper location in IS. After TTCI is built as shown in Figure 1, as an invalidation report, server broadcasts TT and IS.

\subsection{Client-Side Algorithm}

When a client receives an invalidation report, it checks whether the cache's most recent timestamp is within the history window. If it is not within the history window, the entire cache is dropped. Otherwise, the client starts the look-up process from the root node in TT to reach the updated data IDs. Finally, the client validates its cache with the updated information in IS.

In this example, let the last time it was validated be $T^{C}=17$, the timestamp of the current TTCI be $T=32$, invalidation broadcast window be $w=2$, and the

Table 1. Sample Data and OLIST

(a) Updated Data with Timestamps

\begin{tabular}{|c||c|c|c|c|c|c|c|c|c|c|c|c|c|c|c|c|c|c|c|c|}
\hline Data ID & $d 1$ & $d 2$ & $d 3$ & $d 4$ & $d 5$ & $d 6$ & $d 7$ & $d 8$ & $d 9$ & $d 10$ & $d 11$ & $d 12$ & $d 13$ & $d 14$ & $d 15$ & $d 16$ & $d 17$ & $d 18$ & $d 19$ & $d 20$ \\
\hline Timestamp & 24 & 16 & 10 & 6 & 22 & 18 & 26 & 32 & 2 & 20 & 14 & 30 & 8 & 4 & 12 & 28 & 8 & 14 & 24 & 20 \\
\hline
\end{tabular}

(b) OLIST

\begin{tabular}{|c||c|c|c|c|c|c|c|c|c|c|c|c|c|c|c|c|}
\hline Timestamp & 2 & 4 & 6 & 8 & 10 & 12 & 14 & 16 & 18 & 20 & 22 & 24 & 26 & 28 & 30 & 32 \\
\hline Data ID & $d 9$ & $d 14$ & $d 4$ & $d 13, d 17$ & $d 3$ & $d 15$ & $d 11, d 18$ & $d 2$ & $d 6$ & $d 10, d 26$ & $d 5$ & $d 1, d 19$ & $d 7$ & $d 16$ & $d 12$ & $d 8$ \\
\hline
\end{tabular}




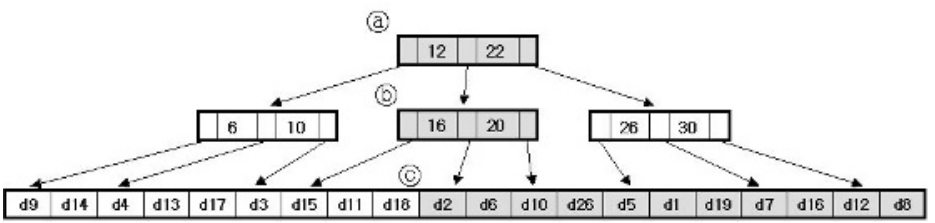

Fig. 1. TTCI

IR broadcast interval be $L=5$. Then it is within the history window. Starting from root node $a$, the client reaches position $c$ in IS via node $b$. Figure 1 shows the TTCI for this example while the client uses only the shaded nodes.

\section{Performance Analysis}

We present analysis of our scheme by comparing to DRCI 3 and TS[1]. We address the invalidation report size as a performance metric, becuase it directly affects the consumption of scarce bandwidth and limited resources of mobile devices. The other is the cache usage ratio. Invalidation reports aim to maximize the cache utilization. Thus the number of valid data in cache after the validation reflects the effectiveness of an invalidation report. We define the cache usage ratio as the following equation 1 , where NumOfCachedData denote the entire size of the cache, and NumOfValidData denote the number of currently cached data.

$$
\text { CacheUsageRatio }=\frac{\text { NumOfValidData }}{\text { NumOfCachedData }} \times 100(\%)
$$

As the update rate increases, the IR size of DRCI and TTCI increases because they should contain more updated information. In all ranges, TTCI outperforms DRCI with an average improvement of more than 25\%. Additionally, we can predict that TTCI will also perform better on tuning time, because it uses smaller IRs and supports selective tuning. Cache usage ratio degrades with increasing update rate. However, even under $50 \%$ of the update rate, TTCI substantially outperforms DRCI and TS, proving that it is less sensitive to the update rate. Thus, as expected, TTCI efficiently copes with various update rate.

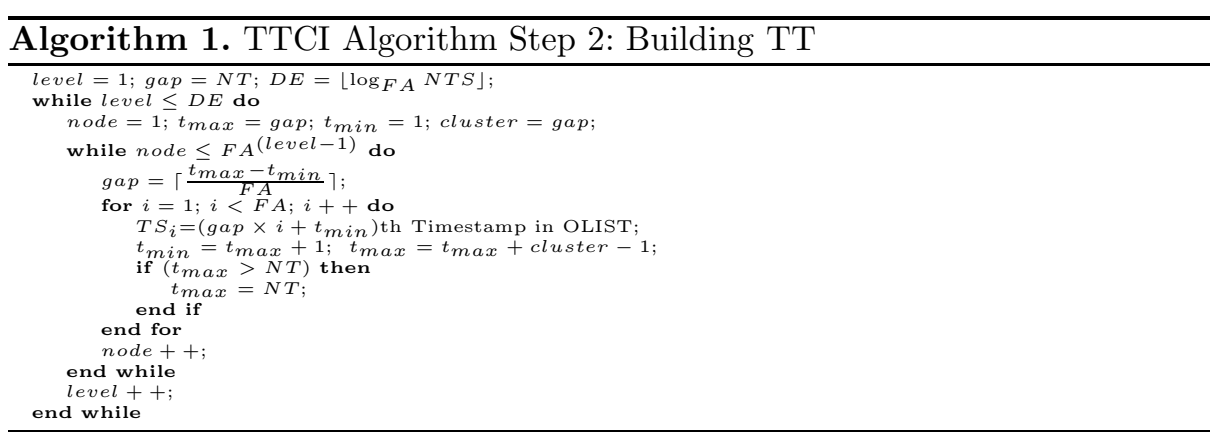




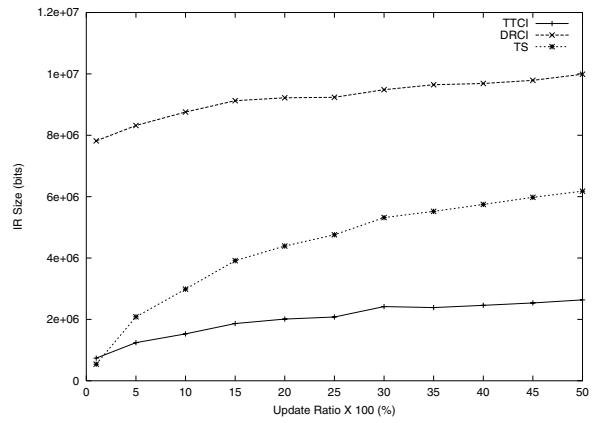

(a) Effect on the invalidation report size

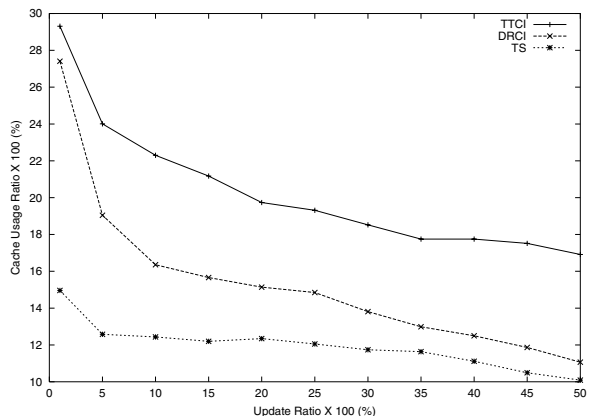

(b) Effect on the cache usage ratio

Fig. 2. Simualtion Results

\section{Conclusion}

In this paper, we have explored the problems of existing cache invalidation schemes. As the update rate increases, existing cache invalidation schemes suffer from a large invalidation report size and low cache utilizations. Furthermore, most cache-level cache invalidation schemes do not support selective tuning, which is important for the battery power conservation. We have proposed a new cache invalidation report scheme, called TTCI to cope with the above problems. TTCI dynamically organizes the updated data IDs into groups using a timestamp tree. As a result, the invalidation report size of TTCI becomes less sensitive to increasing update rate. TTCI also supports selective tuning while it performs cache-level invalidation. We have analyzed the performance of our TTCI scheme against existing schemes. We have verified the effectiveness of our TTCI scheme through in-depth simulation experiments.

\section{References}

1. D. Barbara and T. Imielinski, "Sleepers and Workaholics: Caching in Mobile Distributed Environments",Proc. ACM SIGMOD Int. Conf. on Management of Data, pp. 1-12, 1994.

2. S-Y. Yi, W. Song, S. Jung, and S. Park, "A Cost Effective Cache Consistency Method for Mobile Clients in Wireless Environment",DASFAA 2004, pp. 908-915, 2004 .

3. K-L. Tan, B.C. Ooi, "An Evaluation of Cache Invalidation Strategies in Wireless Environments",IEEE Transactions on parallel and Distributed systems, vol.12, no.8, pp. 789-807, 2001.

4. A. Elmagarmid, J. Jing, A. (Sumi) Helal, And C. Lee, "Scalable Cache Invalidation Algorithms for Mobile Data Access",IEEE Transactions on knowledge and data engineering, vol 15, no 6, pp. 1498-1511 2003. 